\title{
Otimização linear na harmonização de pratos e vinhos regionais do Vale do São
}

\section{Francisco}

\author{
Linear optimization in the harmonization of regional dishes and wines from the São Francisco \\ Valley \\ Optimización lineal en la armonización de platos regionales y vinos del Valle de São Francisco
}

Recebido: 20/01/2022 | Revisado: 28/01/2022 | Aceito: 30/01/2022 | Publicado: 31/01/2022

Raquel Amorim da Silva
ORCID: https://orcid.org/0000-0002-8912-1292
Instituto Federal de Educação, Ciência e Tecnologia do Sertão Pernambucano, Brasil
E-mail: raquel.amorim @ aluno.ifsertao-pe.edu.br
Alysson Livio Vasconcelos Guedes
ORCID: https://orcid.org/0000-0003-4001-3853
E-mail:alysso.livio@ifsertao-pe.edu.br
Islaine Santos Silva
Instituto Federal de Educação, Ciência e Tecnologia do Sertão Pernambucano, Brasil
ORCID: https://orcid.org/0000-0002-1821-9014
E-mail: islaine.santos@ifsertano-pe.edu.br
Elis Tatiane da Silva Nogueira
ORCItituto Federal de Educação, Ciência e Tecnologia ho Sttps://orcid.org/0000-0002-9111-4665
ORstituto Federal de Educação, Ciência e Tecnologia do Sertão Pernambucano, Brasil
E-mail: elis.nogueira@ ifsertao-pe.edu.br

\begin{abstract}
Resumo
A junção entre vinho e comida é denominada harmonização e, esta prática proporciona a combinação dos sabores, favorecendo contemplar alimentos e bebidas com maior equilíbrio, sem que os sabores se sobreponham, desta forma garantindo apreciar as principais características de ambos. O presente trabalho tem como objetivo aplicar a otimização linear na harmonização de pratos e vinhos regionais do Submédio Vale do São Francisco. Foi realizado o levantamento de oito vinhos e dez pratos, buscando-se avaliar as relações entre algumas comidas e vinhos da região. Atribuiu-se notas de 1 a 5 para as harmonizações, classificando-as como 'sem harmonia', 'refresco', 'neutro', 'boa harmonização' e 'correspondência sinérgica', supondo-se a situação em que cada prato e cada vinho só poderia ser harmonizado uma vez, sem repetição. Para isto, utilizou-se uma ferramenta de otimização matemática para obter um resultado ótimo para uma situação com mais de um milhão de possibilidades diferentes de escolha. Os resultados para o problema da melhor escolha entre vinho e comida obedecem às regras de harmonização existentes, tornando-a mais eficiente e mostrando que a aplicação matemática tem a possibilidade de resolver um problema real.
\end{abstract}

Palavras-chave: Vinhos tropicais; Gastronomia; Programação linear; Combinação.

\begin{abstract}
The junction between wine and food is called harmonization and this practice provides the combination of flavors, favoring the contemplation of foods and drinks with greater balance, without the flavors overlapping, thus ensuring to appreciate the main characteristics of both. The present work aims to apply linear optimization in the harmonization of regional dishes and wines from the Submédio Vale do São Francisco. A survey of eight wines and ten dishes was carried out, seeking to evaluate the relationships between some foods and wines in the region. Grades from 1 to 5 were assigned to the harmonies, classifying them as 'no harmony', 'refreshment', 'neutral', 'good harmonization' and 'synergistic correspondence', assuming the situation in which each dish and each wine could only be paired once, without repetition. For this, a mathematical optimization tool was used to obtain an optimal result for a situation with more than a million different possibilities of choice. The results for the problem of the best choice between wine and food obey the existing rules of harmonization, making it more efficient and showing that the mathematical application has the possibility of solving a real problem.
\end{abstract}

Keywords: Tropical wines; Gastronomy; Linear programming; Combination.

\section{Resumen}

La unión entre el vino y la comida se llama armonización y esta práctica proporciona la combinación de sabores, favoreciendo la contemplación de alimentos y bebidas con mayor equilibrio, sin que los sabores se superpongan, 
Research, Society and Development, v. 11, n. 2, e45211225964, 2022

(CC BY 4.0) | ISSN 2525-3409 | DOI: http://dx.doi.org/10.33448/rsd-v11i2.25964

asegurando así apreciar las principales características de ambos. El presente trabajo tiene como objetivo aplicar la optimización lineal en la armonización de platos regionales y vinos del Sub-medio Valle de São Francisco.Se realizó un relevamiento de ocho vinos y diez platos, buscando evaluar las relaciones entre algunos alimentos y vinos de la región. Se asignaron notas del 1 al 5 a las armonías, clasificándolas como 'sin armonía', 'refrescante', 'neutra', 'buena armonización' y 'correspondencia sinérgica', asumiendo la situación en la que cada plato y cada vino sólo podía ser emparejado una vez, sin repetición. Para ello se utilizó una herramienta de optimización matemática para obtener un resultado óptimo para una situación con más de un millón de posibilidades de elección diferentes. Los resultados para el problema de la mejor elección entre vino y comida obedecen a las reglas de armonización existentes, haciéndolo más eficiente y mostrando que la aplicación matemática tiene la posibilidad de resolver un problema real.

Palabras clave: Vinos tropicales; Gastronomía; Programación lineal; Combinación.

\section{Introdução}

A associação entre comida e vinho não é considerada uma prática recente, pois historicamente foi citada na bíblia, como na passagem da última ceia de Jesus (Manfio, 2019). A junção entre vinho e comida é denominada harmonização e, esta prática proporciona a combinação dos sabores, favorecendo contemplar alimentos e bebidas com maior equilíbrio sem que os sabores se sobreponham, desta forma garantindo apreciar as principais características de ambos (Novakoski \& Freire, 2012).

É necessário observar algumas regras para proporcionar um casamento perfeito entre o vinho com os alimentos, para que as características de ambos sejam apreciadas (Pimentel, 2019). Para que essa combinação aconteça de forma satisfatória é necessário observar fatores como, escolha dos tipos de vinho; as características sensoriais da bebida; as particularidades do prato (Borges, 2007).

A harmonização poderá ser realizada por duas formas, a primeira por semelhança, que consiste em buscar características semelhantes tanto no vinho quanto na comida para que no final haja uma perfeita harmonia de aromas, texturas e sabores (Spence, 2020). A segunda forma é por contraste, esta harmonização é caracterizada pela escolha de vinho e comida com atributos diferentes afim de obter um melhor equilíbrio (Spence et al., 2017).

Devido à grande diversidade de pratos e vinhos encontrados atualmente no Brasil pode tornar-se difícil realizar a melhor junção entre a bebida e a comida. De acordo com Santos (2017) para garantir uma refeição prazerosa devido à grande pluralidade, é indicado realizar a associação de comida e vinho de uma mesma região.

A região do Submédio Vale do São Francisco localizada no nordeste brasileiro destaca-se na produção de vinhos finos (Pereira, 2013) entre brancos, tintos e espumantes. Além disso o local dispõe de uma gastronomia típica do sertão, considerado um importante patrimônio no contexto turístico (Gimenes, 2006).

De acordo com Santos (2017) o Brasil está caminhando a passos lentos com relação à referências ligadas à harmonização, principalmente quando pensamos em vinhos e pratos de diversas regiões do país. Desta forma tem-se buscado constantemente por novas técnicas e procedimentos para ajudar na hora de montar a harmonização entre comida e bebida, de forma que um não interfira no sabor do outro (Santos, 2017).

Como exemplo de um novo procedimento de avaliação surge o modelo matemático de otimização linear, que consiste em minimizar ou maximizar relações de combinação em uma função linear, podendo ser aplicada em diversas situações como exemplo, planejamentos de combinações (Silva, 2002).

A programação linear é uma técnica de otimização utilizada a fim de descobrir o lucro máximo ou o custo mínimo de uma determinada situação com muitas opções de escolha (Prado,2016). Além disso, a modelagem linear pode ajudar com a relação de combinações entre diversos produtos para planejamento de demandas. Existem pesquisas que utilizam desta ferramenta para definir cardápios nutricionais (Belai et al., 2016; Spak, 2017). Nesse contexto, o objetivo do presente estudo foi aplicar a otimização linear na harmonização de pratos e vinhos regionais do Submédio Vale do São Francisco. 


\section{Materiais e Métodos}

\subsection{Fontes de dados}

\subsubsection{Vinhos}

Foram selecionados oito vinhos finos elaborados em vinícolas da região do Submédio Vale do São Francisco, sendo três vinhos tintos, dois brancos e três espumantes um rosé e dois brancos. No Quadro 1 encontram-se os atributos sensoriais gustativos para cada vinho.

Quadro 1 - Levantamentos dos atributos sensoriais gustativos.

\begin{tabular}{|ccc|c|}
\hline & VINHOS & CLASSIFICAÇÃO & CARACTERÍSTICAS SENSORIAIS \\
GUSTATIVAS
\end{tabular}

Fonte: Autores.

\subsubsection{Pratos Regionais}

Foram realizadas a seleção de dez pratos (feijoada, dobradinha, caldo de mocotó, acarajé, ensopado de surubim, moqueca de peixe, bolo de rolo, cartola, caldo de abóbora com carne seca e baião de dois) da culinária local para montar a harmonização com os vinhos selecionados. O Quadro 2 apresenta as características de sabores dos pratos.

Quadro 2 - Comida típica regional e suas características.

\begin{tabular}{|c|c|c|}
\hline COMIDAS REGIONAIS & CARACTERÍSTICAS DE SABORES & CARACTERÍSTICAS DO PRATO \\
\hline Feijoada & Alto teor de gordura & $\begin{array}{c}\text { Feijão preto, carne suína, bovina e } \\
\text { linguiça. }\end{array}$ \\
\hline Dobradinha & Sabor expressivo e gorduroso & $\begin{array}{c}\text { Feijão branco, bucho de boi e } \\
\text { especiarias. }\end{array}$ \\
\hline Caldo de mocotó & Untuoso e expressivo & Pé de boi e temperos verdes. \\
\hline Acarajé & Gorduroso e apimentado & $\begin{array}{l}\text { Feijão fradinho, azeite de dendê, } \\
\text { vatapá, camarão e tomate. }\end{array}$ \\
\hline Ensopado de surubim & Leve & $\begin{array}{l}\text { Filé de surubim, leite de coco e } \\
\text { temperos à gosto. }\end{array}$ \\
\hline Moqueca de peixe & Rico em sabor e untuosidade & Peixe, leite de coco, azeite de dendê \\
\hline Bolo de rolo & Adocicado & $\begin{array}{c}\text { Massa de pão de ló, açúcar e } \\
\text { goiabada. }\end{array}$ \\
\hline Cartola & Doce e gorduroso & Queijo, banana e canela em pó. \\
\hline Caldo de abóbora com carne seca & Adocicado e gorduroso & $\begin{array}{c}\text { Abóbora, carne seca, linguiça e cheiro } \\
\text { verde. }\end{array}$ \\
\hline Baião de dois & untuoso & $\begin{array}{l}\text { Feijão de corda, arroz, bacon e queijo } \\
\text { coalho. }\end{array}$ \\
\hline
\end{tabular}




\subsection{Modelagem do problema}

Observando as regras de harmonização podemos fazer escolhas seguras sobre qual vinho deve combinar com um determinado prato. No entanto, foi criado a situação onde haverá exclusividade de combinações, ou seja, cada prato irá ser escolhido com apenas um vinho, sem repetições dos vinhos ou dos pratos. Nesse problema são 8 vinhos e 10 pratos. O primeiro vinho pode ser pareado com qualquer um dos 10 pratos, o segundo vinho pode ser pareado com qualquer um dos 9 pratos restantes e assim sucessivamente. Então conta-se 10x9x8x7x6x5x4x3 e o resultado é 1.814 .400 (um milhão, oitocentos e catorze mil e quatrocentas) possibilidades diferentes de resposta conforme Figura 1. Portanto foi aplicado a otimização matemática para escolher a combinação de pratos e vinhos que maximize a harmonização entre eles de acordo com Maculun (2006).

A programação Linear Inteira pertence a uma área de ciência matemática relacionada à otimização combinatória (ou otimização matemática), onde algumas ou todas as variáveis possuem valores inteiros. Nesta situação de harmonização enquadra-se na categoria de Problema de Afectação ou Problema de Alocação (em inglês, Assignment Problem).

Figura 1. Representação do problema de alocação entre vinhos e pratos.

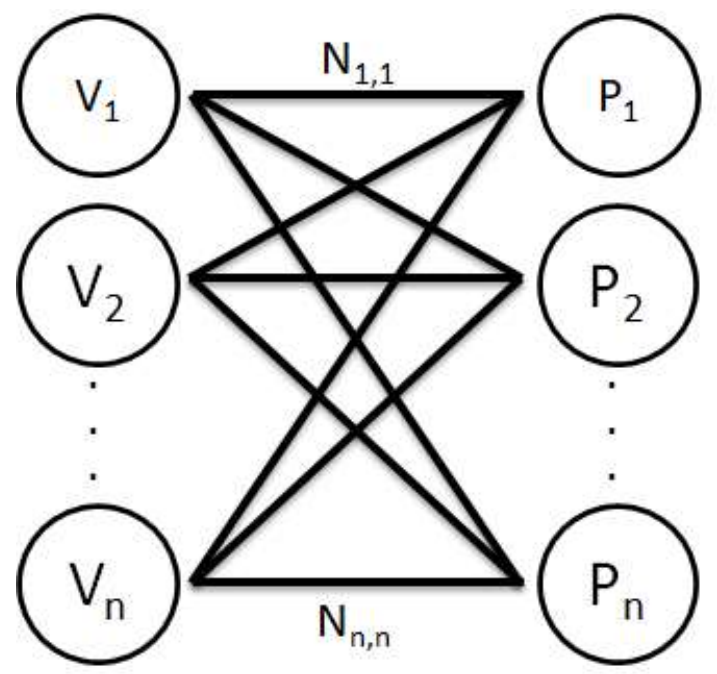

$V_{i}-$ Vinho i; $P_{j}$ - Prato j; $N_{i j}$ - Nota da harmonização entre o vinho i e o prato j. Sendo i,j =1, 2, ... n. Fonte: Autores.

Buscou-se a combinação entre pratos e vinhos que a soma das notas das harmonizações seja máxima. Portanto devemos maximizar a seguinte Função Objetivo:

$$
\text { Equação 1: } \quad f_{o b j}=\sum_{i=1}^{n} \sum_{j=1}^{n} N_{i j} x_{i j}
$$


Sujeito as seguintes restrições:

$$
\begin{aligned}
& x_{i j}=0 \text { ou } 1 \quad \forall i, j \in\{1,2, \ldots n\} \\
& \sum_{i=1}^{n} x_{i j}=1 \quad \forall i=1,2, \ldots \text { ou } n \\
& \sum_{j=1}^{n} x_{i j}=1 \quad \forall i=1,2, \ldots \text { ou } n
\end{aligned}
$$

Somou-se todas as notas e multiplicou-se esses valores por 0 ou 1 . Se $\mathrm{x}_{\mathrm{ij}}$ vale 1 a nota entre o vinho $\mathrm{i}$ e prato $\mathrm{j}$ deve estar presente e 0 caso contrário. As somas de $\mathrm{x}_{\mathrm{ij}}$ devem valer 1 indicando que foi escolhido apenas uma combinação para cada vinho e prato.

As análises foram feitas no software R (R Core Team ${ }^{\circledR}$, 2021) através do pacote IpSolve (Michel Berkelaar and others, 2020).

\subsection{Atribuição de notas}

O Livro Food and wine pairing, a sensory experience (Harrington, 2008), em tradução livre "Harmonização de comida e vinho, uma experiência sensorial”, classifica as harmonizações em: sem harmonia, refresco, neutro, boa harmonia e harmonia sinérgica. Foi adaptado as classificações tornando-as notas de 1 a 5 , a fim de utilizá-las ao modelo matemático. Uma nota baixa, como 1 ou 2 não significa que é uma má experiência, mas sim que foi encontrado um efeito de harmonização mais sinérgico. Seria importante colocar aqui então que quanto maior a nota, mais sinergia entre a combinação. No Quadro 3 expressa os códigos usados para os vinhos e pratos.

Quadro 3. Identificação dos vinhos e pratos.

\begin{tabular}{|cc|}
\hline VINHOS & PRATOS \\
\hline V1 - Vinho tinto - Syrah & P1 - Feijoada \\
V2 - Vinho branco - Verdejo & P2 - Dobradinha \\
V3 - Espumante blanc de blanc moscatel - Moscato Canelli & P3 - Caldo de mocotó \\
V4 - Vinho tinto - Touriga Nacional, Carbenet Sauvignon, Syrah e Malbec; & P4 - Acarajé \\
V5 - Vinho branco - chenin blanc e viognier & P5 - Ensopado de Surubim \\
V6 - Espumante brut rosé - Syrah & P6 - Moqueca de peixe \\
V7 - Espumante demi-sec Branco - Syrah e Moscato Canelli & P7 - Bolo de rolo \\
& P8 - Cartola \\
\hline
\end{tabular}

Fonte: Autores. 


\section{Sem harmonia (Nota 1)}

Nessa classificação o vinho e a comida quando são consumidos juntos tem um efeito negativo no paladar. Um exemplo disso é o surubim, peixe de carne macia e sabor leve, harmonizado com o vinho tinto V4, um vinho estruturado que harmoniza bem com carnes vermelhas. Portanto com essa diferença de características do prato e do vinho o efeito é totalmente negativo nessa situação.

\section{Refresco (Nota 2)}

Nessa classificação o vinho torna-se coadjuvante na refeição. Servindo como um "limpa-paladar”, para pratos com um certo teor de gordura. Um exemplo disso é o caldo de mocotó que é um prato com sabor untuoso e expressivo, harmonizado com o vinho branco V2, que é um vinho fresco e com boa acidez, não se obtém a melhor combinação porque o vinho serviria melhor para um prato mais leve.

\section{Neutro (Nota 3)}

Uma harmonização neutra geralmente ocorre com vinhos "bom para qualquer situação", ou seja, é o famoso vinho coringa. No vale do São Francisco, região quente, de clima tropical semiárido, os vinhos para essa classificação seria os espumantes e os vinhos brancos, pois eles têm uma boa acidez que combina com praticamente tudo, frituras, saladas, peixes, entre outros. Um exemplo de harmonização neutra é a sobremesa cartola, que é feita com banana, queijo e açúcar, sendo harmonizado com o espumante V6, que é um vinho leve e refrescante, o que ajuda a limpar o paladar da gordura da sobremesa, casaria bem nessa situação.

\section{Boa harmonização (Nota 4)}

Uma boa harmonização ocorre quando regras básicas de harmonização são obedecidas, de modo que a única coisa que não faria esse prato subir de nível, seria pequenos detalhes como intensidade de sabor, ou seja, o vinho e o prato precisam está na mesma intensidade. Um exemplo de boa harmonização é o caldo de abóbora com carne seca que é um prato com sabor pronunciado e adocicado por conta da abóbora, sendo harmonizado com o espumante V7 que é um vinho leve e agradável ao paladar, mas não tem a mesma intensidade que o prato precisa, portanto por conta desse detalhe essa combinação se encaixou como boa harmonização.

\section{Correspondência sinérgica (Nota 5)}

Nessa classificação estão as combinações que mais se adequam e se completam formando uma perfeita sinergia. Além disso, essa combinação entre vinho e comida gera uma experiência completamente nova.

Um exemplo disso é a feijoada que é um prato com grande intensidade de sabores e um alto teor de gordura harmonizado com o vinho tinto V1 que é um vinho com boa acidez e presença de taninos, casa perfeitamente com o prato.

A Tabela 1 apresenta as notas sobre as combinações dos pratos e vinhos com base nos conceitos de harmonizações por semelhança e contraste tendo em consideração as características de sabores da bebida e comida. 
Research, Society and Development, v. 11, n. 2, e45211225964, 2022

(CC BY 4.0) | ISSN 2525-3409 | DOI: http://dx.doi.org/10.33448/rsd-v11i2.25964

Tabela 1 - Notas de combinações de vinho e comida usando as características de sabores.

\begin{tabular}{cccccccccccc}
\hline & P1 & P2 & P3 & P4 & P5 & P6 & P7 & P8 & P9 & P10 \\
\hline V1 & 5 & 5 & 5 & 1 & 1 & 1 & 1 & 1 & 1 & 2 \\
V2 & 2 & 2 & 2 & 3 & 5 & 5 & 1 & 1 & 4 & 3 \\
V3 & 2 & 2 & 2 & 2 & 1 & 1 & 5 & 3 & 1 \\
V4 & 5 & 5 & 5 & 1 & 1 & 1 & 1 & 1 & 1 & 1 \\
V5 & 2 & 2 & 2 & 2 & 5 & 5 & 1 & 1 & 2 & 4 \\
V6 & 2 & 2 & 2 & 5 & 3 & 4 & 1 & 3 & 5 & 1 \\
V7 & 2 & 2 & 2 & 2 & 3 & 4 & 4 & 5 & 4 & 1 \\
V8 & 5 & 5 & 5 & 1 & 1 & 1 & 1 & & 1 & 5 \\
\hline
\end{tabular}

Vinhos: V1 - Vinho tinto - Syrah; V2 - Vinho branco - Verdejo; V3 - Espumante blanc de blanc moscatel - Moscato Canelli; V4 - Vinho tinto - Touriga Nacional, Carbenet Sauvignon, Syrah e Malbec; V5 - Vinho branco - chenin blanc e viognier; V6 - Espumante brut rosé Syrah; V7 - Espumante demi-sec Branco - Syrah e Moscato Canelli; V8 - Vinho tinto - Grenache, Shiraz e Mourvèdre. Pratos: P1 Feijoada; P2 - Dobradinha; P3 - Caldo de mocotó; P4 - Acarajé; P5 - Ensopado de Surubim; P6 - Moqueca de peixe; P7 - Bolo de rolo; P8 - Cartola; P9 - Caldo de Abobora com Carne Seca; P10 - Baião de dois. Fonte: Autores.

\section{Resultados e Discussão}

A solução do problema levou a um conjunto de combinações, maximizando a soma das notas das harmonizações. Foi utilizado combinação de 1 para 1, ou seja, o vinho é degustado com apenas 1 prato, sem repetições. Dessa maneira 2 pratos ficaram de fora, a dobradinha e o acarajé, pois foi escolhido mais pratos do que vinhos no modelo. O que também não quer dizer que não há boas harmonizações desses pratos com os vinhos apresentados, mas que eles ficaram de fora para que fosse obtido uma soma de notas máxima no resultado final. Na Figura 2 analisa-se o resultado obtido através da otimização linear, observando os pratos regionais que se ligam harmoniosamente aos vinhos produzidos no Submédio do Vale do São Francisco. 
Research, Society and Development, v. 11, n. 2, e45211225964, 2022

(CC BY 4.0) | ISSN 2525-3409 | DOI: http://dx.doi.org/10.33448/rsd-v11i2.25964

Figura 2. Relação de harmonização de vinho e comida através da otimização linear.

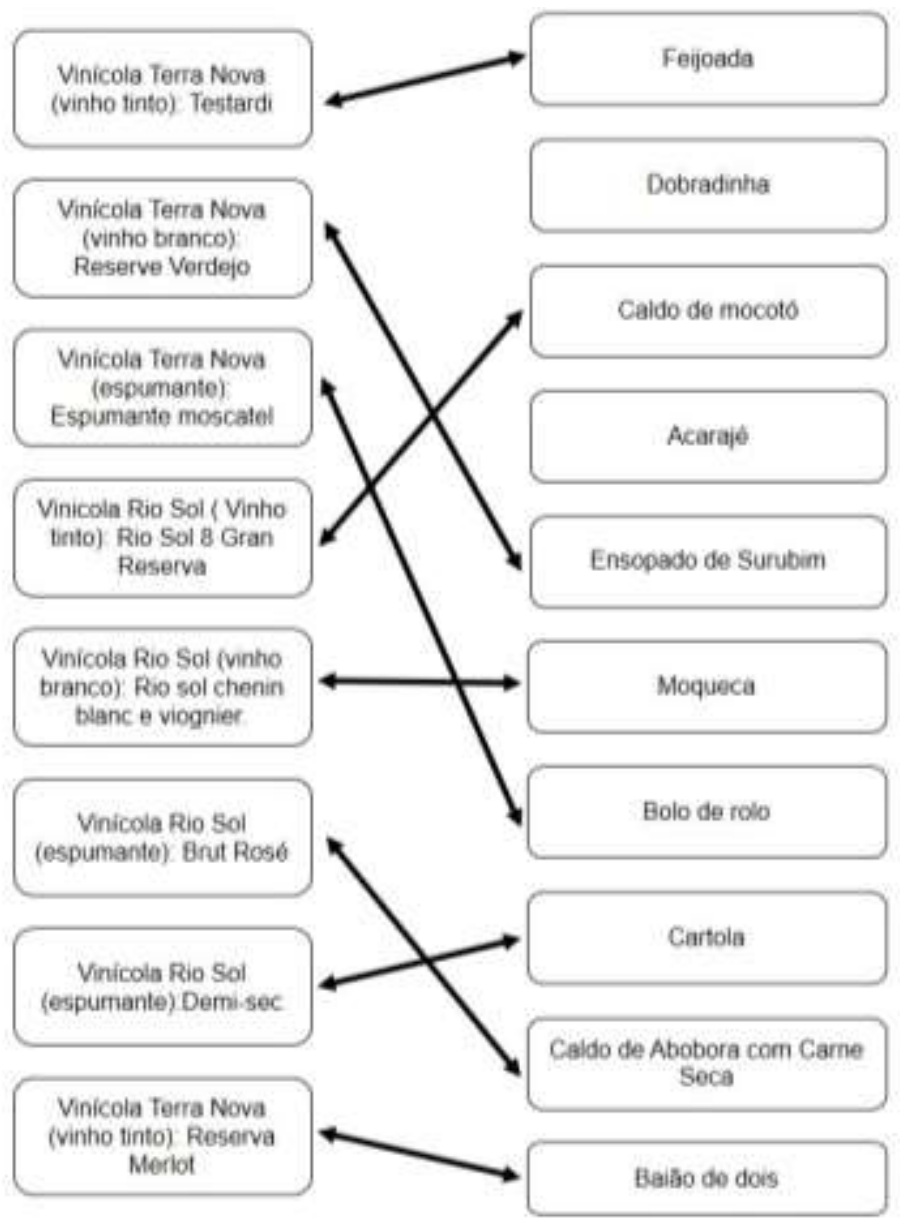

Fonte: Autores.

A feijoada é um prato com grande intensidade de sabores e um alto teor de gordura. $\mathrm{O}$ vinho escolhido para esse prato, precisa ter uma boa acidez e presença tânica, pois os taninos da bebida deixam o prato mais leve e fácil de ser digerido. Assim como a acidez também ajuda no equilíbrio do prato. Portanto o vinho escolhido foi vinho tinto V1.

Na sequência tem-se o ensopado de surubim que tem a carne branca, macia e sabor equilibrado. O vinho escolhido para esse prato, foi o vinho branco V2 de acidez equilibrada e bastante floral. De acordo com Lecat e Chapuis (2017) não importa o preparo do peixe, sendo grelhado, cozido ou frito pode ser acompanhado de vinho branco.

O bolo de rolo, por sua vez, que é tradicionalmente recheado com goiabada e feito de pão de ló. O vinho escolhido para essa sobremesa precisa ser adocicado para que case perfeitamente com a doçura da goiabada, logo a escolha foi o vinho espumante V3 equilibrando doce/doce. Nesta situação a harmonização foi realizada por similaridade, ou seja, possui sabores e perfil semelhantes (Eschevins et al., 2018).

Outra harmonização definida foi o caldo de mocotó que é um prato com sabor untuoso e expressivo. O vinho escolhido para esse prato precisa ter uma boa presença tânica, pois os taninos têm o poder de limpar a gordura (Galmarini et al., 2016). Desse modo o vinho escolhido foi o vinho tinto V4, por possuir uma maior carga de taninos.

Outro prato harmonizado foi moqueca de peixe, um prato bastante untuoso devido à gordura presente. A regra tradicional de harmonização diz que vinho branco deve ser servido com peixe (Buodo et al., 2019), mas com o passar dos tempos percebe-se a necessidade de pensar também nos componentes da bebida devido à preparação do prato, como a acidez. 
Para esta combinação foi escolhido o vinho branco V5, a qual apresenta alta acidez onde vai ajudar na limpeza das papilas gustativas devido a gordura do prato.

Posteriormente vem a carne seca que tem um certo teor de gordura e sabor pronunciado, combinando bem com a doçura da abóbora. O vinho escolhido para esse prato precisa ter médio corpo e ser fresco para ajudar a limpar o paladar. Dessa maneira a escolha foi o vinho V6.

Já a cartola é uma sobremesa que é feita com banana, queijo e açúcar. O vinho escolhido para esse prato precisa ter uma acidez equilibrada, pois o vinho precisa limpar a gordura do queijo e equilibrar o açúcar da sobremesa. Assim a escolha foi o espumante V7, por apresentar acidez e as borbulhas oriundas do gás carbônico, estes elementos, possuem a ação de neutralização da gordura (Freire, 2018).

Por último o baião de dois que é um prato untuoso e muito saboroso. O vinho escolhido para esse prato precisa ser um vinho com boa acidez, pois a acidez irá ajudar a contrapor a gordura do prato. Dessa forma o vinho escolhido foi o vinho tinto V8. A acidez do vinho ajuda a digerir a gordura tornando o alimento macio (Harrington, 2008).

\section{Conclusão}

A harmonização de comida e vinho é realmente grandiosa e cheia de possibilidades de combinações para todos os tipos e gostos, nesse estudo, o direcional são para os pratos e vinhos da região do Vale do Submédio São Francisco, rico em sabores e vinhos de ótima qualidade.

A pesquisa teve como motivação desmistificar a harmonização, solucionando um problema real do cotidiano dos consumidores de vinho, e diante disso, percebeu-se que a modelagem matemática pode auxiliar no processo de tomada de decisão e na resolução de problemas.

O estudo de novas interações da enogastronomia é crucial para o desenvolvimento da enologia como um todo, pois é necessário evoluir constantemente para entregar cada vez mais produtos de qualidade e experiências enogastronômicas inovadoras. Por se tratar de uma ciência complexa que utiliza de várias outras áreas de estudo como, biologia, química, física, matemática, e diversas outras para processar bons resultados, a enologia deve unificar laços cada vez mais com outras ramificações de conhecimentos. É necessário realizar mais estudos de aplicação da otimização linear aplicados a outros pratos e vinhos da região.

\section{Referências}

Belai, D. R., Silva, P. P., da Silva, E. G., \& da Luz, L. P. (2016). Sistema especialista de elaboração de cardápios nutricionais/Expert System for Nutrition Managemen. TECHNO REVIEW. International Technology, Science and Society Review, 5(2), 179-184.

Berkelaar, M. (2015). Package 'lpSolve’.

Borges, E. P. (2007). Harmonização: o livro definitivo do casamento do vinho com a comida. Mauad Editora Ltda.

Buodo, G., Rumiati, R., Lotto, L., \& Sarlo, M. (2019). Os pares de comida e bebida afetam o processamento do apetite dos sinais alimentares com diferentes propriedades recompensadoras? Evidência de medidas subjetivas, comportamentais e neurais. Food Quality and Preference , 75 , $124-132$.

Eschevins, A., Giboreau, A., Allard, T., \& Dacremont, C. (2018). O papel da semelhança aromática no emparelhamento de alimentos e bebidas. Qualidade e preferência alimentar, $65,18-27$.

Ferreira, M. D. S. (2018). Harmonização de vinhos finos com preparações veganas (Bachelor's thesis, Brasil).

Galmarini, MV, Loiseau, AL, Visalli, M., \& Schlich, P. (2016). Uso da dominância temporal das sensações (TDS) multi-ingestão para avaliar a influência do queijo na percepção do vinho. Journal of Food Science, 81 (10), S2566-S2577.

Gimenes, M. H. S. G. (2006). Patrimônio Gastronômico, Patrimônio Turístico: uma reflexão introdutória sobre a valorização das comidas tradicionais pelo IPHAN e a atividade turística no Brasil. Seminário de pesquisa em turismo no Mercosul, 4, 1-15.

Harrington, R. J. (2008). Food and wine pairing, a sensory experience John Wiley \& Sons. Inc., Hoboken. 
Research, Society and Development, v. 11, n. 2, e45211225964, 2022

(CC BY 4.0) | ISSN 2525-3409 | DOI: http://dx.doi.org/10.33448/rsd-v11i2.25964

Lecat, B., \& Chapuis, C. (2017). Harmonização de comida e vinho na Borgonha: O caso dos Grands Crus. Bebidas, 3 (1), 10.

Maculan, N., \& Fampa, M. H. C. (2006). Otimização linear. Editora Universidade de Brasilia: Brasilia.

Manfio, V. (2019). A articulação entre turismo, vinho e a gastronomia na região da Campanha Gaúcha, Brasil. Ágora, 21(1), 35-41.

Michel Berkelaar and others (2020). lpSolve: Interface to 'Lp_solve' v. 5.5 to Solve Linear/Integer Programs. R package version 5.6.15. https://CRAN.Rproject.org/package $=$ lpSolve

Novakoski, D.; \&Freire, R. (2012). Enogastronomia: a arte de harmonizar cardápios e vinhos. Senac.

Pimentel, D. D. (2019). Harmonização de cervejas artesanais pernambucanas com as sobremesas reconhecidas patrimônios culturais imateriais do Estado (Bachelor's thesis, Brasil). Jackson, M. Guia ilustrado Zahar: cerveja. (2a ed.), Zahar.

Prado, D. (2016). Programação linear. Falconi Editora.

R Core Team (2021). R: A language and environment for statistical computing. R Foundation for Statistical Computing, Vienna, Austria. URL https://www.Rproject.org/.

Santos, M. D. S. D. (2017). A enogastronomia no Brasil e a interferência da temperatura na harmonização entre vinhos e preparações.

da Silva, C. T. L. (2002). Problemas de Otimização Linear Canalizados e Esparsos (Doctoral dissertation, Dissertaçao de Mestrado, Instituto de Ciências Matemáticas e de Computaçao-ICMC-USP, Sao Carlos-SP).

Spak, M. D. S. (2017). Aplicação da modelagem matemática para o planejamento de cardápios para restaurantes universitários.

Spence, C. (2020). Combinação de sabores de alimentos e bebidas: uma revisão crítica da literatura. Food Research International, 133 , 109124.

Spence, C., Wang, Q. J., \& Youssef, J. (2017). Combinando sabores e a ordem temporal da degustação. Sabor, 6(1), 1-15. 